\title{
Superpartners at LHC and future colliders: predictions from constrained compactified M-theory
}

\author{
Sebastian A.R. Ellis, Gordon L. Kane and Bob Zheng \\ Department of Physics, University of Michigan, \\ 50 Church St., Ann Arbor, MI 48109, U.S.A. \\ E-mail: sarellis@umich.edu, gkane@umich.edu, byzheng@umich.edu
}

AbStRact: We study a realistic top-down M-theory compactification with low-scale effective Supersymmetry, consistent with phenomenological constraints. A combination of top-down and generic phenomenological constraints fix the spectrum. Three and only three superpartner channels, $\tilde{g} \tilde{g}, \chi_{2}^{0} \chi_{1}^{ \pm}$and $\chi_{1}^{+} \chi_{1}^{-}$(where $\chi_{2}^{0}, \chi_{1}^{ \pm}$are Wino-like), are expected to be observable at LHC-14. We also investigate the prospects of finding heavy squarks and Higgsinos at future colliders. Gluino-stop-top, gluino-sbottom-bottom associated production and first generation squark associated production should be observable at a $100 \mathrm{TeV}$ collider, along with direct production of heavy Higgsinos. Within this framework the discovery of a single sparticle is sufficient to determine uniquely the SUSY spectrum, yielding a number of concrete testable predictions for LHC-14 and future colliders, and determination of $M_{3 / 2}$ and thereby other fundamental quantities.

Keywords: Supersymmetry Phenomenology, Strings and branes phenomenology

ARXIV EPRINT: 1408.1961 


\section{Contents}

1 Introduction 1

2 Theoretical framework $\quad 3$

2.1 Imposing constraints: EWSB and the Higgs mass 4

2.2 Obtaining a benchmark spectrum 4

$\begin{array}{lll}3 & \text { The spectrum and the branching ratios } & 6\end{array}$

$\begin{array}{llr}4 & \text { LHC-14 predictions } & 8\end{array}$

5 Future collider predictions $\quad 10$

6 Conclusion 11

A Moduli stabilization and SUSY breaking in the $G_{2}$-MSSM 12

B Constraints from EWSB and $M_{h} \quad 14$

C Threshold corrections from Kaluza-Klein states 16

\section{Introduction}

If Supersymmetry (SUSY) is considered as an effective theory of a well-motivated ultraviolet (UV) completion, concrete predictions can be made about the Supersymmetric particle (sparticle) spectrum that is to be expected at the $\mathrm{TeV}$ scale. In a top-down approach, the Supersymmetry breaking parameters that in a bottom-up model would be free are instead set by high-scale dynamics. This leads to concrete, testable predictions for sparticle masses, as opposed to treating sparticle masses as free parameters in a multidimensional parameter space.

In this note, we illustrate the power of this top-down approach for a particularly wellmotivated UV completion of Supersymmetry: M-theory compactifications on manifolds with $G_{2}$ holonomy [1-5]. We call this framework the $G_{2}$-MSSM, following earlier papers which have studied its phenomenological properties [6-9]. Due to the rigidity of topdown theoretical constraints from moduli stabilization and Supersymmetry breaking, the sparticle spectrum is completely fixed by electroweak symmetry breaking (EWSB), once both the gravitino mass $M_{3 / 2}$ and the superpotential $\mu$-term are specified. Furthermore, the measured value of the Higgs mass picks out a particular slice in the $\left(\mu, M_{3 / 2}\right)$ plane; thus all sparticle masses can be fixed by determining either $\mu$ or $M_{3 / 2}$.

Given minimal assumptions regarding the geometry of the compact $G_{2}$-manifold, $M_{3 / 2}$ is approximately calculable once the moduli are stabilized and SUSY is broken. This 
results in a central value of $M_{3 / 2}=35 \mathrm{TeV}$. Imposing EWSB and Higgs mass constraints, $M_{3 / 2}=35 \mathrm{TeV}$ corresponds to a gluino mass of $1.5 \mathrm{TeV}$. The result $M_{3 / 2}=35 \mathrm{TeV}$ can be altered if UV threshold corrections to the non-perturbative superpotential are nonnegligible. Allowing a wider range of $20 \mathrm{TeV}<M_{3 / 2}<70 \mathrm{TeV}$ due to currently unknown UV dynamics, in particular the mechanism for generating the $\mu$ parameter, gives a range of predicted gluino masses, $1 \mathrm{TeV}<M_{\text {gluino }}<3 \mathrm{TeV}$.

For concreteness we will focus on a benchmark point with value $M_{3 / 2}=35 \mathrm{TeV}$, which gives a benchmark spectrum with a $1.5 \mathrm{TeV}$ gluino. All sfermion and heavy Higgs masses are of $\mathcal{O}\left(M_{3 / 2}\right)$, while the Wino(Bino)-like lighter gauginos have masses of $615(450) \mathrm{GeV}$. The hierarchy between gauginos and $M_{3 / 2}$ follows from the dynamics of moduli stabilization. Specifically, both the hidden sector meson and moduli F-terms contribute to the gravitino mass $M_{3 / 2}$, while only the moduli F-terms contribute to gaugino masses. The moduli F-terms are suppressed with respect to hidden sector meson F-terms by about $\alpha_{\mathrm{GUT}} \approx 1 / 25$, resulting in $\lesssim \mathrm{TeV}$ gaugino masses despite $M_{3 / 2}$ being tens of $\mathrm{TeV}$ [8]. As will be discussed below, this benchmark spectrum is not constrained by LHC-8. Both gluino pair production and direct electroweak gaugino production should yield discoveries with $\lesssim 300 \mathrm{fb}^{-1}$ of LHC-14 data, particularly since electroweak gauginos yield distinctive signatures through $\chi_{2}^{0} \rightarrow \chi_{1}^{0}+h$ which has a nearly $100 \%$ branching ratio. This bencmark spectrum predicts $\mu \approx 1.4 \mathrm{TeV}$, leading to heavy Higgsinos which are out of reach of LHC-14.

We will also discuss implications of the $G_{2}$-MSSM for future colliders. Taking the benchmark spectrum, we will show that $1.5 \mathrm{TeV}$ Higgsinos are accessible at both 50 and $100 \mathrm{TeV}$ colliders. Furthermore, the heavier squarks are also accessible at $100 \mathrm{TeV}$ colliders, with hundreds of squark-gluino associated production events expected with $\gtrsim 1000 \mathrm{fb}^{-1}$ of data. In particular, we point out for the first time (as far as we know) that for a gluino mass of $\sim \mathrm{TeV}$, the cross-section for associated stop-gluino-top production $p p \rightarrow \tilde{g}+\tilde{t}_{1}+t$, and potentially even sbottom-gluino-bottom $p p \rightarrow \tilde{g}+\tilde{b}_{1}+b$, can be sizeable at $100 \mathrm{TeV}$ colliders for stops and sbottoms lighter than $\sim 20 \mathrm{TeV}$. This is especially relevant for MSUGRA-like theories with universal scalar masses at the GUT scale, in which third generation squarks are expected to be lighter due to RGE effects.

Thus the benchmark spectrum has the remarkable feature that Higgsinos and squarks are accessible at future colliders, despite the scale of SUSY breaking $M_{3 / 2}$ being in the tens of TeV range. Furthermore, the constrained relationship between SUSY breaking parameters implies that within the $G_{2}$-MSSM, the discovery of a single sparticle is enough to actually measure $M_{3 / 2}$. Given the discovery of a single sparticle, the rest of the $G_{2}$-MSSM spectrum is determined uniquely, resulting in a multitude of additional predictions which can be readily confirmed or falsified in ongoing and upcoming collider experiments. This point highlights the potential power of top-down approaches in greatly reducing the naive parameter space of Supersymmetry. We will provide details concerning these points in later work; the results can basically be seen from equations (2.1), (2.2) and figure 1 in section 2 below.

The paper is organised as follows. Section 2 gives a brief overview of the $G_{2}$-MSSM framework, and discusses how the benchmark spectrum corresponding to $M_{3 / 2}=35 \mathrm{TeV}$ is obtained. Readers interested only in experimental predictions should skip this section. Section 3 provides the sparticle spectrum and relevant branching ratios corresponding to the 
benchmark spectrum obtained in section 2. Predictions for LHC-14 are given in section 4, while predictions for future colliders are given in section 5. Finally, we summarise the results in section 6 .

\section{Theoretical framework}

We begin by reviewing some features and successes of the compactified M-theory framework. $G_{2}$-compactifications of M-theory provide a natural setting for full moduli stabilisation and broken $\mathcal{N}=1$ Supersymmetry in a deSitter vacuum, while also solving the gauge hierarchy problem [6-9]. Additionally, EDM [10, 11] and flavour constraints (such as $\left.B_{s} \rightarrow \mu \mu\right)$ [12] are avoided. However, it may not explain $(g-2)_{\mu}$, while the strong CP problem is solved by the axionic components of moduli fields [13]. The presence of late-decaying moduli results in a non-thermal cosmological history which solves the moduli problem and the gravitino problem [14]. Since the baryon asymmetry and the dark matter both arise from moduli decay (including axions), the ratio of baryonic matter to dark matter is calculable [15]. R-parity conservation is expected [16]; thus consistency with the observed DM relic abundance implies that the visible sector LSP will decay to hidden sector DM particles [17]. ${ }^{1} \mu$ is incorporated into the theory following the proposal of Witten [20] and including effects of moduli stabilisation [21]. Calculations in this framework anticipated the mass and decay branching ratios of the Higgs boson observed at the LHC [22].

A key result of the aforementioned references is that once moduli stabilization breaks Supersymmetry and cancels the vacuum energy at tree level, the relationship between SUSY breaking parameters becomes very constrained. Upon moduli stabilization and SUSY breaking, the soft breaking parameters at the renormalization scale $Q \sim M_{\mathrm{GUT}}$ are given by [9]:

$$
\begin{aligned}
& m_{0}^{2} \approx M_{3 / 2}^{2}(1-C), A_{0}^{2} \approx 1.5 M_{3 / 2}(1-C), \\
& M_{a} \approx\left[-0.032 \eta+\alpha_{\mathrm{GUT}}\left(0.034\left(3 C_{a}-C_{a}^{\prime}\right)+0.079 C_{a}^{\prime}(1-C)\right)\right] \times M_{3 / 2}
\end{aligned}
$$

where $C_{a}=(0,2,3)$ and $C_{a}^{\prime}=(33 / 5,7,6) . m_{0}$ and $A_{0}$ are universal soft scalar masses and trilinears, and " $C$ " parameterizes higher order Kähler potential corrections arising from higher dimensional operators as defined in appendix A. The quantity $\eta$ parameterizes KK-threshold corrections to the unified gauge coupling; we will argue in section 2.2 that $\eta \sim 1$, unless the geometry of the $G_{2}$ manifold becomes incredibly complicated. A review of moduli stabilization and SUSY breaking along with the derivation of (2.1) is presented in appendix A. The hierarchy between gaugino masses and $M_{3 / 2}$ arises because $M_{3 / 2}$ feels contributions from both hidden sector meson and moduli F-terms, while $M_{a}$ feels contrbutions from only the moduli $\mathrm{F}$-terms which are suppressed by approximately $\alpha_{\mathrm{GUT}} \approx 1 / 25$ with respect to the meson F-terms. A more detailed discussion of this point is deferred to appendix A.

Thus the entire sparticle spectrum is essentially fixed once $M_{3 / 2}, \mu$ and $C$ are specified. We will show in section 2.1 that imposing consistent EWSB along with the measured value

\footnotetext{
${ }^{1}$ For non-thermal cosmologies, a stable Bino-LSP will overclose the universe, while a stable Wino-LSP is in tension with indirect detection contraints $[18,19]$.
} 
of the Higgs mass $M_{h}=125.2 \pm 0.4 \mathrm{GeV}[23,24]$ reduces the $3 \mathrm{D}$ space of allowed $\left(M_{3 / 2}, \mu, C\right)$ values to an approximately one-dimensional space. As a result, the entire sparticle spectrum is completely determined for a given value of $M_{3 / 2}$. In section 2.2 we will use top-down considerations to approximately calculate $M_{3 / 2}$, giving a central value of $M_{3 / 2} \approx 35 \mathrm{TeV}$ which we use to obtain the benchmark spectrum considered in section 3 and onwards.

\subsection{Imposing constraints: EWSB and the Higgs mass}

In the previous section, we have stated that the sparticle spectrum is essentially determined by three quantities: $M_{3 / 2}, \mu, C$, or equivalently $M_{3 / 2}, \mu / M_{3 / 2}, C$. In principle, these quantities are calculable from the full UV theory. In practice however, there are theoretical uncertainties which preclude a full top-down calculation. Instead, we will show in this section how bottom-up constraints of EWSB along with the measured Higgs mass provide two independent constraints, reducing the naive $3 \mathrm{D}$ region to a one-dimensional strip. This illustrates the power of combining top-down calculations with known bottom-up constraints to increase the predictiveness of a particular theory.

A detailed discussion of how the constraints from EWSB and $M_{h}=125.2 \pm 0.4 \mathrm{GeV}$ are imposed is given in appendix B; the result is shown in figure 1 . One can see that EWSB constraints restrict the region to an approximately 2D-slice. Imposing the constraint $M_{h}=125.2 \pm 0.4$ reduces this slice to a thin band; the thickness of this band is due primarily to experimental uncertainties on $M_{h}, M_{t}$ and $\alpha_{s}$ [22]. The range $0<\mu / M_{3 / 2} \lesssim 0.1$ is motivated by both top-down [21] and little hierarchy [25] arguments; this is discussed in more detail in appendix B.

Upon imposing these bottom-up constraints, the entire sparticle spectrum becomes an approximately one-dimensional strip in the original 3D space. In the next section, we take the perspective that figure 1 fixes the entire spectrum in terms of $M_{3 / 2}$, and use equation (A.6) to approximately compute $M_{3 / 2}$ to be $\approx 35 \mathrm{TeV}$, and obtain the associated benchmark spectrum.

\subsection{Obtaining a benchmark spectrum}

Having established that the entire Supersymmetric particle spectrum is essentially determined for a given value of $M_{3 / 2}$, we discuss how $M_{3 / 2}$ can be approximately computed from the UV theory. Upon moduli stabilization and SUSY breaking, the standard Supergravity expression for the gravitino mass gives [9]:

$$
M_{3 / 2} \approx \frac{9 \times 10^{5}}{V_{X}^{3 / 2}}\left(\frac{A_{2}}{Q}\right) \mathrm{TeV}
$$

where $Q$ is the rank of a hidden sector $\mathrm{SU}(Q)$ gauge group which undergoes gaugino condensation, and $A_{2}$ is the corresponding non-perturbative superpotential coefficient (see eq. (A.1) in appendix A). $V_{X}$ is the volume of the $G_{2}$-manifold in 11-D Planck units. We refer interested readers to appendix A for a more detailed derivation of eq. (2.2).

In principle, $V_{X}$ is a function of the moduli fields and is thus calculable once moduli are stabilized. However the particular expression for $V_{X}$ in terms of moduli fields is not 

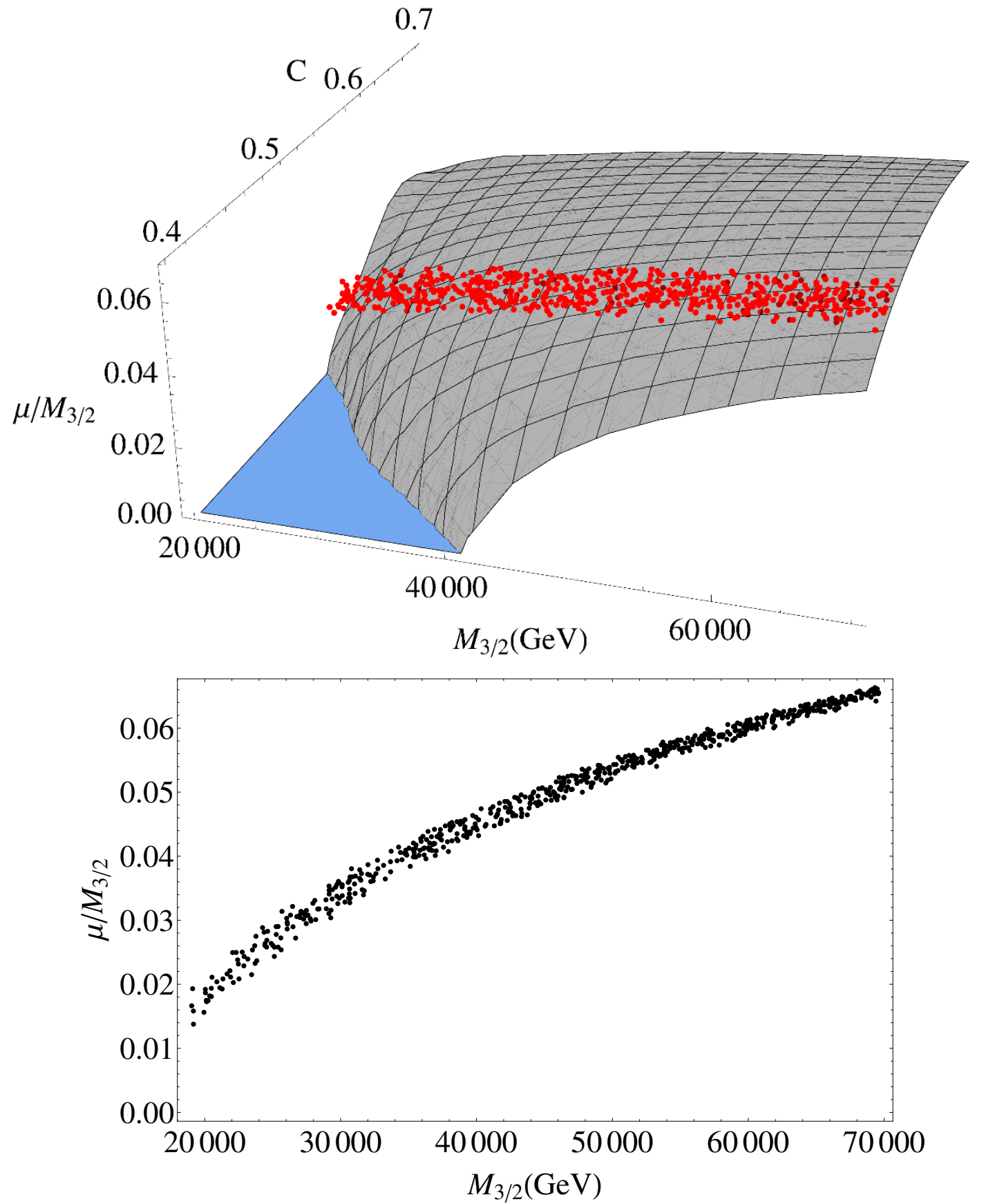

Figure 1. Upper figure: the dark gray surface shows the slice of $M_{3 / 2}, c, \mu / M_{3 / 2}$ parameter space which satisfies EWSB, while red points also satisfy the Higgs mass constraint $M_{h}=125.2 \pm 0.4 \mathrm{GeV}$. The blue shaded region corresponds to points which are inconsistent with $\mu / M_{3 / 2} \lesssim 0.1$; see the appendix for further discussion. In these plots, $\mu$ is defined at the renormalization scale $Q^{2}=$ $m_{\tilde{t}_{1}} m_{\tilde{t}_{2}}$. The mesh lines are added for perspective, and do not have any physical significance. Lower figure: a projection of the upper figure onto the $M_{3 / 2}-\mu / M_{3 / 2}$ plane. 
fully known; we can instead fix $V_{X}$ by ensuring that dimensional reduction to $4 \mathrm{D}$ gives the correct value for Newton's constant [26]. This fixes $V_{X}$ to be [9]:

$$
V_{X} \approx 137.4 L(\mathcal{Q})^{2 / 3}
$$

where $L(\mathcal{Q})$ is a topological invariant which parameterizes threshold corrections from Kaluza-Klein states [26]. The particular value for $L(\mathcal{Q})$ is determined by the embedding of Witten's doublet-triplet splitting mechanism [20] within the compactified M-theory framework. The simplest variant of this mechanism predicts $L(\mathcal{Q})=16$; this is discussed in more detail in appendix $\mathrm{C}$.

Taking these values for our benchmark spectrum, we obtain from (2.2), (2.3):

$$
M_{3 / 2} \approx 35\left(\frac{A_{2}}{Q}\right) \mathrm{TeV}
$$

Note that this also fixes $\eta$ in (A.7) [26]:

$$
\eta=1-\frac{5 \alpha_{\mathrm{GUT}}}{2 \pi} \ln \left(\frac{L(Q)}{q}\right) \approx 0.956
$$

We see that $1-\eta$ is loop suppressed, and can only be $\mathcal{O}(1)$ for $q \gtrsim 100$. This validates our earlier claim that one naturally expects $\eta \sim 1$.

The only remaining undetermined factor in our benchmark spectrum is $A_{2} / Q$ in (2.4). In the context of Supersymmetric field theories, the precise normalization $A_{2}=Q[27]$ in the $\overline{D R}$ scheme was determined by requiring the consistency of various techniques used to study non-perturbative SUSY gauge theories [28]. However, in the present context there may be UV threshold corrections which modify the relation $A_{2} / Q=1$. To obtain the benchmark spectrum, we retain $A_{2} / Q=1$, which gives $M_{3 / 2}=35 \mathrm{TeV}$. Imposing constraints from EWSB and $M_{h}$ for this benchmark value fixes $C \approx 0.52$ and $\mu \approx 1.4$, resulting in the benchmark spectrum discussed in section 3. Figure 2 illustrates the effect of relaxing this assumption; the gluino mass is plotted for $0.6 \lesssim A_{2} / Q \lesssim 1.4$, corresponding to $20 \mathrm{TeV} \lesssim$ $M_{3 / 2} \lesssim 50 \mathrm{TeV} . M_{3 / 2}=20 \mathrm{TeV}$ may barely avoid tension with BBN constraints [29], depending on the particular values of moduli couplings and $T_{R H}$.

In order to obtain a very approximate lower bound on $M_{\tilde{g}}$, we use the FastLim [30] package. The program currently only implements some of the possible event topologies, and does not yet implement cascade decays such as $\tilde{g} \rightarrow q q \chi_{2}^{0} \rightarrow q q h \chi_{1}^{0}$. Therefore, when using FastLim we treat the aforementioned cascade decays as $\tilde{g} \rightarrow q q \chi_{2}^{0} \rightarrow q q \chi_{1}^{0}$, neglecting additional objects from $\chi_{2}^{0} \rightarrow \chi_{1}^{0}+h$. This simplification obviously reduces sensitivity to searches involving high $(\geq 6)$ jet multiplicity. Using this method, we obtain the approximate bound $M_{\tilde{g}} \gtrsim 1.1 \mathrm{TeV}$ in the compactified M-theory framework, though clearly a more precise analysis is desirable. Nonetheless, the benchmark spectrum with a $1.5 \mathrm{TeV}$ gluino mass sits comfortably outside the excluded region, as we will discuss in section 3 .

\section{The spectrum and the branching ratios}

In this section we present the benchmark spectrum that results from the theoretical framework presented in section 2. In the compactified M-Theory all scalars are generically of 


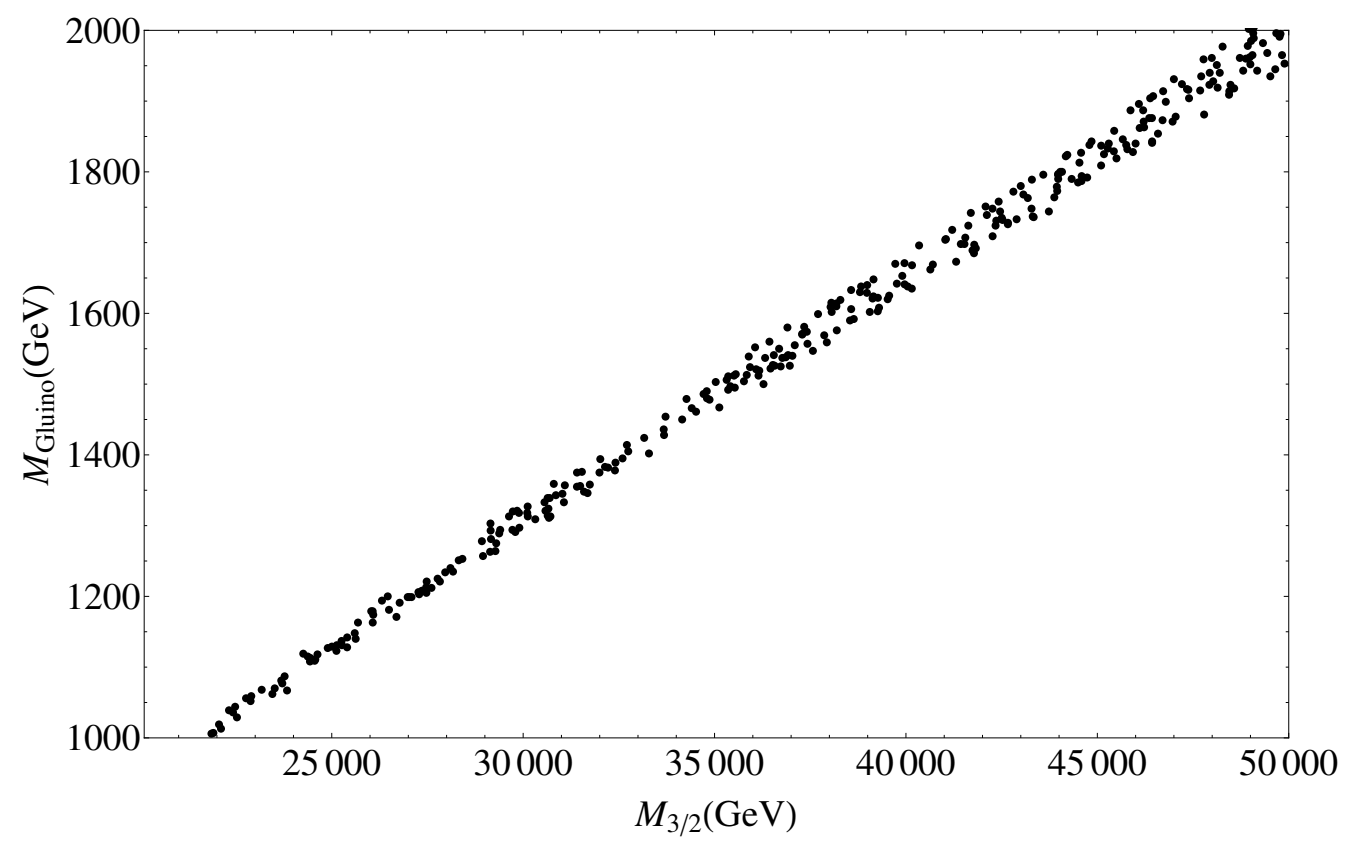

Figure 2. Gluino mass vs $M_{3 / 2}$ for points which satisfy EWSB and Higgs mass constraints, i.e. the red points in figure 1.

order the gravitino mass, with the universal scalar mass given by $m_{0} \sim \mathcal{O}(1) M_{3 / 2}$. We use the computing package SOFTSUSY [31] to do two-loop RGE evolution of the high scale soft parameters to obtain sparticle pole masses.

We compute the branching ratios for the decays of some of the superpartners using SDECAY [32], which are given in table 1. We focus in particular on the superpartners that we expect to see at the LHC or at future colliders.

Since the squark masses are of $\mathcal{O}\left(M_{3 / 2}\right)$ at the high scale, they are not detectable at the LHC. RGE running splits the squarks to give the physical spectrum shown in figure 3. The neutralinos $\chi_{1}^{0}\left(\chi_{2}^{0}\right)$ are Bino (Wino)-like, while $\chi_{3}^{0}, \chi_{4}^{0}$ are Higgsino-like. Note that the mixing angles in the neutralino and chargino sectors are small, as $\mu \gg M_{W}, M_{Z}$. In previous studies of the $G_{2}$-MSSM, the LSP was taken to be Wino-like, as a Wino-LSP serves as a good DM candidate in string-motivated non-thermal cosmologies [33]. However, obtaining a Wino-like as opposed to a Bino-like LSP requires large KK-threshold corrections to GUT scale gaugino masses (see e.g. [34]), which we argued is unnatural in section 2. In this note, we assume that any would-be Bino-like LSP relic abundance decays to a hidden sector DM candidate, but the LSP is sufficiently long-lived to appear stable on collider scales. ${ }^{2}$

Of note is the $100 \%$ branching ratio of $\chi_{1}^{ \pm} \rightarrow \chi_{1}^{0} W^{ \pm}$, and the $98.7 \%$ branching ratio of $\chi_{2}^{0} \rightarrow \chi_{1}^{0} h$. Note that the $\chi_{2}^{0} \rightarrow \chi_{1}^{0} Z$ decay width is subdominant to that of $\chi_{2}^{0} \rightarrow \chi_{1}^{0} h$. This can be explained as follows. The $\chi_{2}^{0} \chi_{1}^{0} h$ coupling arises from couplings of the form $\tilde{W}^{0} \tilde{H} h$ and $\tilde{B}^{0} \tilde{H} h$ in the gauge eigenstate basis. Since $\chi_{1}^{0}$ and $\chi_{2}^{0}$ are Bino and Winolike, the $\chi_{2}^{0} \rightarrow \chi_{1}^{0} h$ amplitude is suppressed by a single power of gaugino-Higgsino mixing

\footnotetext{
${ }^{2}$ This occurs for example if $\chi_{1}^{0}$ decays to hidden sector DM via kinetic mixing through a $Z^{\prime}$, with $M_{Z^{\prime}} \sim \mathcal{O}\left(M_{3 / 2}\right)[17]$.
} 


\begin{tabular}{|c|c|c|c|c|c|}
\hline & & Decay & $\mathrm{BR}(\%)$ & & \\
\hline & & $\chi_{4}^{0} \rightarrow \chi_{1}^{ \pm} W^{\mp}$ & 60 & & \\
\hline & & $\chi_{4}^{0} \rightarrow \chi_{2}^{0} h$ & 27 & & \\
\hline Decay & BR (\%) & $\chi_{4}^{0} \rightarrow \chi_{1}^{0} h$ & 8 & & \\
\hline$\tilde{g} \rightarrow \chi_{1}^{+} q_{1,2} \bar{q}_{1,2}$ & 25 & $\chi_{4}^{0} \rightarrow \chi_{2}^{0} Z$ & 4 & Decay & BR (\%) \\
\hline$\tilde{g} \rightarrow \chi_{1}^{ \pm} b \bar{t}, t \bar{b}$ & 23 & $\chi_{4}^{0} \rightarrow \chi_{1}^{0} Z$ & 2 & $\chi_{2}^{ \pm} \rightarrow \chi_{1}^{ \pm} h$ & 31 \\
\hline$\tilde{g} \rightarrow \chi_{1}^{0} t \bar{t}$ & 20 & & & $\chi_{2}^{ \pm} \rightarrow \chi_{1}^{ \pm} Z$ & 30 \\
\hline$\tilde{g} \rightarrow \chi_{2}^{0} q_{1,2} \bar{q}_{1,2}$ & 12 & $\chi_{3}^{0} \rightarrow \chi_{1}^{ \pm} W^{\mp}$ & 60 & $\chi_{2}^{ \pm} \rightarrow \chi_{2}^{0} W^{ \pm}$ & 30 \\
\hline$\tilde{g} \rightarrow \chi_{1}^{0} q_{1,2} \bar{q}_{1,2}$ & 8 & $\chi_{3}^{0} \rightarrow \chi_{2}^{0} Z$ & 26 & $\chi_{2}^{ \pm} \rightarrow \chi_{1}^{0} W^{ \pm}$ & 9 \\
\hline$\tilde{g} \rightarrow \chi_{2}^{0} b \bar{b}$ & 7 & $\chi_{3}^{0} \rightarrow \chi_{1}^{0} Z$ & 8 & & \\
\hline$\tilde{g} \rightarrow \chi_{2}^{0} t \bar{t}$ & 4 & $\chi_{3}^{0} \rightarrow \chi_{2}^{0} h$ & 4 & $\chi_{1}^{ \pm} \rightarrow \chi_{1}^{0} W^{ \pm}$ & 100 \\
\hline$\tilde{g} \rightarrow \chi_{1}^{0} b \bar{b}$ & 1 & $\chi_{3}^{0} \rightarrow \chi_{1}^{0} h$ & 2 & & \\
\hline & & $\chi_{2}^{0} \rightarrow \chi_{1}^{0} h$ & 98 & & \\
\hline & & $\chi_{2}^{0} \rightarrow \chi_{1}^{0} Z$ & 2 & & \\
\hline
\end{tabular}

Table 1. Branching ratios of gluino, neutralinos and charginos. The numbers don't add to 100 in the case of the $\chi_{4}^{0}$ branching ratios due to rounding errors.

angles, $\mathcal{O}\left(M_{Z} / \mu\right)$. In contrast, in the gauge eigenstate basis only Higgsinos couple directly to $Z$ via couplings of the form $Z_{\mu} \tilde{H}^{\dagger} \bar{\sigma}^{\mu} \tilde{H}$. Thus the $Z_{\mu} \chi_{1}^{0 \dagger} \bar{\sigma}^{\mu} \chi_{2}^{0}$ coupling is suppressed by two powers of gaugino-Higgsino mixing angles, resulting in a suppresion of the $\chi_{2}^{0} \rightarrow \chi_{1}^{0} Z$ amplitude by $\mathcal{O}\left(M_{Z}^{2} / \mu^{2}\right)$.

Note that this spectrum with a $1.5 \mathrm{TeV}$ gluino should not have been discovered at LHC8. In simplified models with decoupled squarks, the strongest limits on the gluino production come from multijet + MET searches [35-38], which place the bound $M_{\tilde{g}} \gtrsim 1.35 \mathrm{TeV}$. When setting limits on simplified models, the gluino is assumed to have a $100 \%$ branching ratio into either $q q \chi_{1}^{0}$ or $t t \chi_{1} 0$ final states. For more realistic spectra such as the one considered here, the gluino mass bound will weaken significantly due to branching ratio factors.

\section{$4 \quad$ LHC-14 predictions}

In this section, using the spectrum derived and presented above, we discuss the channels we expect to be observable at the LHC. We expect to see three channels, $p p \rightarrow \tilde{g} \tilde{g}, p p \rightarrow \chi_{2}^{0} \chi_{1}^{ \pm}$ and $p p \rightarrow \chi_{1}^{ \pm} \chi_{1}^{\mp}$, and only these. The fact that only these three channels would be apparent is a feature of the M-theory construction. The scalars being heavy makes them kinematically inaccessible. The hierarchy between $\mu$ and the gaugino masses $M_{a}$ (and $\mu \gg M_{Z}$ ) results in a Bino-like LSP and Wino-like NLSP with heavy Higgsinos, meaning that only two neutralino/chargino direct production channels are accessible at LHC-14. 


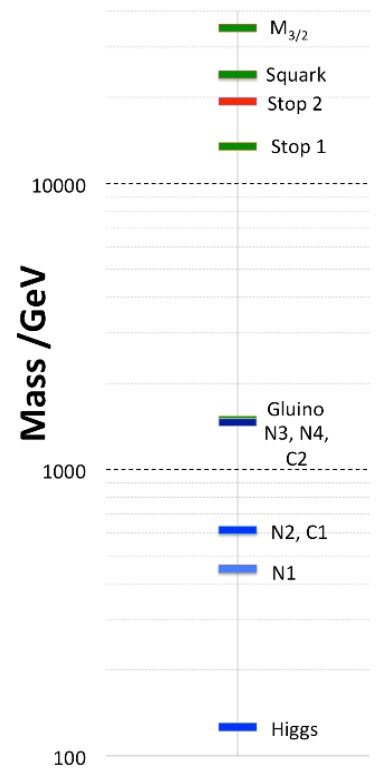

\begin{tabular}{|l|c|}
\hline Particle & Mass $(\mathrm{GeV})$ \\
\hline$m_{0}$ & 24200 \\
$M_{3 / 2}$ & 35000 \\
$\tilde{q}_{L, R}$ & 24000 \\
$\tilde{t}_{2}$ & 19300 \\
$\tilde{t}_{1}$ & 13500 \\
$\tilde{b}_{2}$ & 23900 \\
$\tilde{b}_{1}$ & 19300 \\
$\tilde{g}$ & 1500 \\
$\chi_{1}^{0}$ & 450 \\
$\chi_{2}^{0}$ & 614 \\
$\chi_{3}^{0}$ & 1460 \\
$\chi_{4}^{0}$ & 1460 \\
$\chi_{1}^{ \pm}$ & 614 \\
$\chi_{2}^{ \pm}$ & 1460 \\
$h$ & $125.2^{3}$ \\
\hline
\end{tabular}

Figure 3. Spectrum given GUT scale input values calculated from the theory for the central value $M_{3 / 2}=35 \mathrm{TeV}$. This spectrum has the GUT scale inputs $m_{0} \approx 24 \mathrm{TeV}$ and $A_{0} \approx 25 \mathrm{TeV}$, where $m_{0}$ and $A_{0}$ are respectively the universal scalar mass and soft-breaking trilinear. The GUT scale gaugino masses are $M_{1}=-1020 \mathrm{GeV}, M_{2}=-730 \mathrm{GeV}, M_{3}=-590 \mathrm{GeV}$. Details on how this spectrum was derived are presented in section 2. For the gaugino masses and trilinear, we take the sign convention opposite to that of SOFTSUSY [31]. This relative sign affects the 2-loop term in the gaugino mass RGE's which is proportional to $A_{t}$.

We computed using the production cross-sections to leading order for the three channels listed above using MadGraph5 [39] and multiplied them by $\mathrm{K}$ factors calculated using Prospino [40-42]. The results, including the expected number of events $N$ given $300 \mathrm{fb}^{-1}$ of data, are tabulated below.

Given the LSP mass of $450 \mathrm{GeV}$, a $1.5 \mathrm{TeV}$ gluino is expected to be discoverable at the $5 \sigma$ level at LHC-14 given $300 \mathrm{fb}^{-1}$ of data $[43,44]$. The gluino mass and cross-section allow immediate deduction of the gluino spin [45] and therefore confirmation that the discovery is indeed Supersymmetry. With squarks heavy, that procedure is straightforward. Furthermore, the $\chi_{2}^{0} \rightarrow \chi_{1}^{0} h$ decay mode allows for discovery potential in the chargino/neutralino direct production channels. For the benchmark spectrum considered here, direct $\chi_{2}^{0}, \chi_{1}^{ \pm}$ production should be discoverable at LHC-14 with $1000 \mathrm{fb}^{-1}$ of data [46]. We understand that careful background studies need to be done to be sure these processes can be observed. The signatures are distinctive and event numbers large enough so it seems likely signals can be seen, but people more expert than us need to demonstrate the signals are really robust.

\footnotetext{
${ }^{3}$ For details regarding how the Higgs mass is calculated, we refer the reader to appendix B.
} 


\begin{tabular}{|l|c|c|}
\hline Channel & $\sigma(\mathrm{fb})$ & $N$ \\
\hline$p p \rightarrow \tilde{g} \tilde{g}$ & 19 & 5800 \\
$p p \rightarrow \chi_{2}^{0} \chi_{1}^{ \pm}$ & 19 & 5800 \\
$p p \rightarrow \chi_{1}^{ \pm} \chi_{1}^{\mp}$ & 10 & 3000 \\
\hline
\end{tabular}

Table 2. Production cross sections at $\sqrt{s}=14 \mathrm{TeV}$.

\begin{tabular}{|l|c|c|c|c|}
\hline Channel & $\sigma_{50 \mathrm{TeV}}(\mathrm{fb})$ & $N_{50 \mathrm{TeV}}$ & $\sigma_{100 \mathrm{TeV}}(\mathrm{fb})$ & $N_{100 \mathrm{TeV}}$ \\
\hline$p p \rightarrow t \tilde{t}_{1} \tilde{g}$ & $7.1 \times 10^{-5}$ & 0 & $1.6 \times 10^{-2}$ & 47 \\
$p p \rightarrow b \tilde{b}_{1} \tilde{g}$ & $2.6 \times 10^{-6}$ & 0 & $3.0 \times 10^{-3}$ & 9 \\
$p p \rightarrow \tilde{q}_{1(L, R)} \tilde{g}$ & $3.2 \times 10^{-4}$ & 1 & $3.0 \times 10^{-1}$ & 900 \\
$p p \rightarrow \chi_{3}^{0} \chi_{4}^{0}$ & $9.2 \times 10^{-1}$ & 2800 & 3.4 & 10200 \\
$p p \rightarrow \chi_{3}^{0} \chi_{2}^{ \pm}$ & 1.8 & 5400 & 6.4 & 19200 \\
$p p \rightarrow \chi_{4}^{0} \chi_{2}^{ \pm}$ & 1.8 & 5400 & 6.4 & 19200 \\
$p p \rightarrow \chi_{2}^{ \pm} \chi_{2}^{\mp}$ & 1.0 & 3000 & 3.7 & 11100 \\
\hline
\end{tabular}

Table 3. Production cross sections at future colliders.

\section{$5 \quad$ Future collider predictions}

In this section, we briefly discuss the possible discoveries to be made at future colliders given the spectrum under consideration. We focus in particular on two possible protonproton colliders, one with $\sqrt{s}=50 \mathrm{TeV}$, and the other with $\sqrt{s}=100 \mathrm{TeV}$. Prospects for Supersymmetry at such higher energy colliders has been studied recently [44, 47, 48], although not in the context of a top-down, UV complete theory.

Given the spectrum, we find that some crucial new channels are accessible, namely $p p \rightarrow t \tilde{t}_{1} \tilde{g}, p p \rightarrow b \tilde{b}_{1} \tilde{g}, p p \rightarrow \tilde{q}_{1(L, R)} \tilde{g}, p p \rightarrow \chi_{3}^{0} \chi_{4}^{0}, p p \rightarrow \chi_{3}^{0} \chi_{2}^{ \pm}, p p \rightarrow \chi_{4}^{0} \chi_{2}^{ \pm}$and $p p \rightarrow \chi_{2}^{ \pm} \chi_{2}^{\mp}$.

Unfortunately, scalars are too heavy to be pair-produced [47]. However, we note that associated production of first family squarks with gluinos is accessible. The splitting of the light stop and the heavier first family squark masses, plus the kinematic measurement of the first family squarks, combine to give a precise measurement of the gravitino mass, the fundamental quantity that determines all masses in the theory, and thus deeply probes Supersymmetry breaking!

The associated production of stops and sbottoms can come from gluon splitting, or from the top (bottom) quark being considered as a parton. The question of when the top quark parton distribution function (PDF) becomes important has been studied recently [49], and it has been found that using a top PDF has only a small effect at $\sqrt{s}=100 \mathrm{TeV}$. Therefore we simulate the production of stop gluino by looking at diagrams where the gluon splits and emits a top quark also. The bottom PDF is better known, and has a more significant effect at the energies in question, but for the purposes of this calculation, we present results using gluon splitting for the associated production of sbottoms. The dominant Feynman 


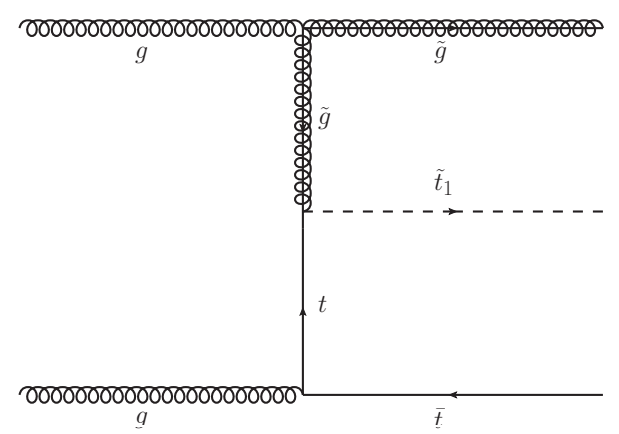

Figure 4. Dominant Feynman graph for stop associated production by gluon splitting.

diagram for top stop gluino production is shown in figure 4 . The diagram for sbottoms is identical with $b, \tilde{b}$ swapped for $t, \tilde{t}$. We recognise that careful studies of bottom and top PDFs need to be done to get fully reliable numbers.

We computed using MadGraph5 [39] the production cross-sections to leading order for these channels for both $\sqrt{s}=50 \mathrm{TeV}$ and $100 \mathrm{TeV}$. The results are tabulated below, including the number of events $N$ expected given $3000 \mathrm{fb}^{-1}$ of data.

Although the number of stop events is not large, the gluino mass will already be known from LHC, which makes the stop and bottom search significantly simpler. The sbottom production cross-section is expected to be greater given that we make the approximation of gluon splitting rather than using the bottom PDF. We expect 15 or so stop events should already be found given $1000 \mathrm{fb}^{-1}$ of data, as well as 300 first generation squark events. The relatively large number of first family squark associated production events means these should be detectable. We also expect the heavy neutralinos and chargino to be detected, already at a $50 \mathrm{TeV}$, and certainly at a $100 \mathrm{TeV}$ collider. Note there are other electroweakino production channels e.g. $\chi_{2}^{0} \chi_{2}^{ \pm}$which have subdominant production crosssections, two or more orders of magnitude smaller than those presented here. Because of their small production cross-sections, we do not list those channels here.

\section{Conclusion}

In this note, we have examined predictions for Supersymmetric particle masses in the $G_{2}$-MSSM, motivated by phenomenogically realistic compactifications of $M$-theory. By combining top-down constraints from moduli stabilization with bottom-up constraints from EWSB and the measured Higgs mass, the sparticle spectrum is completely determined by $M_{3 / 2}$. Furthermore, given reasonable assumptions regarding the topology of the $G_{2}$ manifold, the gravitino mass is approximately calculable, giving a benchmark value $M_{3 / 2}=$ $35 \mathrm{TeV}$. The predicted value for $M_{3 / 2}$ can be further constrained once the UV dynamics which generate the $\mu$ parameter are better understood; see figure 1 .

The benchmark spectrum corresponding to $M_{3 / 2}=35 \mathrm{TeV}$ is not constrained by LHC8, and turns out to provide exciting phenomenology for LHC-14 and future colliders. The gluino mass is expected to be about $1.5 \mathrm{TeV}$ for this benchmark spectrum, while the Wino(Bino)-like gaugino mass is about $614(450) \mathrm{GeV}$. The hierarchy between gaugino 
masses and $M_{3 / 2}$ arises because $M_{3 / 2}$ feels contributions from both the hidden sector meson and moduli F-terms, while gaugino masses only feel contributions from the moduli F-terms which are suppressed by about $\alpha_{\mathrm{GUT}} \approx 1 / 25$ with respect to the meson F-terms. Three and only three production channels should discoverable at LHC-14: $p p \rightarrow \tilde{g} \tilde{g}, p p \rightarrow \chi_{2}^{0} \chi_{1}^{ \pm}$and $p p \rightarrow \chi_{1}^{ \pm} \chi_{1}^{ \pm}$where $\chi_{1}^{0}$ and $\chi_{2}^{0}$ are respectively Bino and Wino-like. The expected signature of the $\chi_{1}^{ \pm} \chi_{1}^{ \pm}$channel is $\chi^{+} \chi^{-} \rightarrow W^{+} W^{-}+$MET. The $\chi_{2}^{0} \chi_{1}^{ \pm}$production channel gives the final state $\chi_{2}^{0} \chi_{1}^{ \pm} \rightarrow W^{ \pm} h+$ MET, which should be quite a clear channel at the LHC [46].

We have also investigated the prospects for the discovery of the heavier stops, first family squarks and Higgsinos at future colliders. We find that associated production of gluino stop $p p \rightarrow \tilde{g} \tilde{t}_{1} t$, gluino sbottom $p p \rightarrow \tilde{g} \tilde{b}_{1} b$ as well as gluino squark production $p p \rightarrow \tilde{g} \tilde{u}, \tilde{d}$ should be seen at a $100 \mathrm{TeV}$ collider, with leading-order production crosssections of $1 \times 10^{-2}, 3 \times 10^{-3}$ and $3 \times 10^{-1} \mathrm{fb}$ respectively. This leads to hundreds of gluino-squark events given $3000 \mathrm{fb}^{-1}$ of data; precise knowledge of the gluino mass can help seperate these events from SM background. The heavy Higgsinos should also be detectable at a $50 \mathrm{TeV}$ collider, and produced in relative abundance at a $100 \mathrm{TeV}$ collider. The relevant Higgsino production channels are $p p \rightarrow \chi_{3}^{0} \chi_{4}^{0}, p p \rightarrow \chi_{3}^{0} \chi_{2}^{ \pm}, p p \rightarrow \chi_{4}^{0} \chi_{2}^{ \pm}$and $p p \rightarrow \chi_{2}^{ \pm} \chi_{2}^{ \pm}$. The relevant production cross sections at 50 (100) TeV are $\sigma \sim 1.8$ (6.4) fb for $p p \rightarrow \chi_{3,4}^{0} \chi_{2}^{ \pm}$, and $\sigma \sim 1.0$ (3.5) fb for $p p \rightarrow \chi_{3}^{0} \chi_{4}^{0}$ and $p p \rightarrow \chi_{2}^{ \pm} \chi_{2}^{ \pm}$. Thus given $3000 \mathrm{fb}^{-1}$ of data, we expect of order a few thousand events for each channel at a $50 \mathrm{TeV}$ collider, and of order tens of thousands of events at a $100 \mathrm{TeV}$ collider.

To summarise, we have shown that the $G_{2}$-MSSM provides a constrained top-down framework, in which gluinos and some electroweakinos are discovered at LHC-14. The discovery of a single sparticle uniquely determines the remainder of the sparticle spectrum. Thus given a discovery of gauginos at LHC-14, a discovery of squarks and Higgsino at $100 \mathrm{TeV}$ colliders within the predicted mass range would give strong evidence towards Supersymmetry and an UV completion like the compactified M-theory construction presented here.

\section{Acknowledgments}

We would like to thank B.S. Acharya, J.R. Ellis, P. Kumar, M. Papucci, A. Pierce and C.P. Yuan for useful discussions. The work of SARE, GLK and BZ is supported in part by Department of Energy grant DE-SC0007859.

\section{A Moduli stabilization and SUSY breaking in the $G_{2}$-MSSM}

In this appendix, we review the relationship between low energy Supersymmetry and UV dynamics within the $G_{2}$-MSSM. This relationship is discussed in $[8,9]$, and was preceded by a detailed study of dynamical Supersymmetry breaking through moduli stabilization $[6,7]$. We will only describe results relevant for our goal of making predictions for Supersymmetric spectra for LHC and future colliders. Interested readers are referred to [50] for a more detailed summary. 
In order to stabilize moduli and obtain a de Sitter vacuum, previous studies have assumed the generic presence of an $\mathrm{SU}(Q) \times \mathrm{SU}(P+1)$ hidden sector, where $\mathrm{SU}(Q)$ is pure super Yang-Mills and $\mathrm{SU}(P+1)$ is super QCD-like with $N_{f}=1$. Non-perturbative effects generate the following superpotential $[27,51]$ which breaks Supersymmetry and stabilizes all moduli:

$$
W=A_{1} \phi^{-2 / P} \exp i b_{1} f_{1}+A_{2} \exp i b_{2} f_{2}
$$

where $2 \pi b_{1}\left(2 \pi b_{2}\right)=P^{-1}\left(Q^{-1}\right)$. Here $f_{1}$ and $f_{2}$ are the gauge kinetic functions of the $\mathrm{SU}(Q)$ and $\mathrm{SU}(P+1)$ gauge groups, and $\phi$ is the $\mathrm{SU}(P+1)$ meson condensate in Planck units. We assume $f_{1} \propto f_{2}$; otherwise it is difficult to stabilize moduli within the supergravity approximation [7]. For consistency with previous works, we make the simplifying assumption $f_{1}=f_{2}=f_{\text {vis }}$, where $f_{\text {vis }}$ is the visible sector gauge kinetic function. We furthermore assume that the visible sector is an SU(5) GUT.

In order to proceed with the moduli stabilization analysis, we use the Kähler potential derived in $[9]$ :

$$
K=-3 \log 4 \pi^{1 / 3} V_{X}+\kappa_{\alpha \beta} \frac{\Phi_{\alpha}^{\dagger} \Phi_{\beta}}{V_{X}}+\frac{\bar{\phi} \phi}{V_{X}}+c_{\alpha \beta} \frac{\bar{\phi} \phi}{V_{X}} \frac{\Phi_{\alpha}^{\dagger} \Phi_{\beta}}{V_{X}}+\ldots
$$

where the volume of the $G_{2}$ manifold, $V_{X}$, is a homogenous function of the moduli of degree $7 / 3$ [52], $\Phi, \Phi^{\dagger}$ represent visible sector fields, $\kappa_{\alpha \beta}$ is the Kähler metric, and “..." represent higher dimensional operators which are neglected in the analysis.

The term proportional to $c_{\alpha \beta}$ is a higher order correction studied in [9]. It induces flavor changing neutral currents unless the elements of $c_{\alpha \beta}$ are all small, or $c_{\alpha \beta} \sim \propto \kappa_{\alpha \beta}$ up to small corrections. Because scalars are heavy and the tree-level CP phases are rotated away, flavor effects are not expected to cause phenomenogical problems [12]. For simplicity, we assume here that the coefficients of $c_{\alpha \beta}$ are diagonal and universal in the basis where $\kappa_{\alpha \beta}$ is diagonal. Thus we take:

$$
c_{\alpha \beta}=\left(\frac{C}{3}\right) \kappa_{\alpha \beta}
$$

The normalization for $C$ in (A.3) follows that of [9]. We will see that $C \neq 0$ is required for consistent EWSB. Relaxing the assumption taken in (A.3) will induce moderate flavor dependence in the soft breaking parameters, but will not change the gravitino mass or the overall sparticle mass scale.

Given (A.1), (A.2) and (A.3), determining the dynamics of moduli stabilization is straightforward, though rather cumbersome and has been carried out in [9]. The gravitino mass is determined by the standard supergravity relation:

$$
\begin{aligned}
M_{3 / 2} & =m_{p l} e^{K / 2}|W| \approx 0.035 V_{X}^{-3 / 2} m_{p l}|Q-P| \frac{A_{2}}{Q} e^{-\left(\frac{P_{\text {eff }}}{Q-P}\right)}, \\
P_{\text {eff }} & =\frac{14(3(Q-P)-2)}{3(3(Q-P)-2 \sqrt{6(Q-P)})}
\end{aligned}
$$

where in the second equality we have fixed moduli at their stabilized values and imposed vanishing vacuum energy [7, 9]. Note that for typical values, $V_{X} \sim \mathcal{O}(730)$ [9]. Imposing 
vanishing of the vacuum energy enforces $Q-P \geq 3$, but does not fix $Q-P$ itself. However, from (A.4) we see that for $Q-P \geq 4$, there still exists a large hierarchy between $M_{3 / 2}$ and the electroweak scale. Thus if a vanishing vacuum energy is imposed, the set of solutions with $Q-P=3$ solves the hierarchy problem. Taking $Q-P=3$ in (A.4) results in:

$$
M_{3 / 2} \approx \frac{9 \times 10^{5}}{V_{X}^{3 / 2}}\left(\frac{A_{2}}{Q}\right) \mathrm{TeV} .
$$

This result was used in section 2.2 to obtain the benchmark value $M_{3 / 2} \approx 35 \mathrm{TeV}$.

Using the standard relations between the Supergravity Lagrangian and soft SUSY breaking parameters [53], it is then straightforward to determine the relation between soft scalar masses, trilinears and $M_{3 / 2}$, as well as the gaugino masses $M_{a}(a=1,2,3)$ at the renormalization scale $Q=M_{\mathrm{GUT}}$ :

$$
m_{0}^{2} \approx M_{3 / 2}^{2}(1-C), A^{0} \approx 1.5 M_{3 / 2}(1-C), \quad M_{a}=\frac{e^{K / 2} F^{i} \partial_{i} f_{\mathrm{vis}}}{2 i \operatorname{Im} f_{\mathrm{vis}}}+M_{a}^{\text {anomaly }}
$$

where $m_{0}$ is the universal scalar soft mass, and we haved define trilinears as $\mathcal{L}=$ $-y_{i j} A \tilde{Q}_{i, L} H_{q} \tilde{q}_{j, R}$. We have seperated the tree-level and anomaly-induced [54] contributions to $M_{a}$. The tree level contribution to $M_{a}$ is proportional to the moduli F-terms $F_{s}$; moduli stabilization results in $F_{s} \propto M_{3 / 2} / V_{Q}$ where $V_{Q} \approx 1 / \alpha_{\text {GUT }}$ is the visible sector 3 -cycle volume [8]. Thus, the tree-level gaugino masses are $M_{a}^{\text {tree }} \sim \alpha_{\mathrm{GUT}} M_{3 / 2}$, and since the anomaly contribution is loop-suppressed, the two contributions are of comparable size. Such a hierarchy between scalars and gaugino masses has also been observed in other corners of string theory $[55,56]$, though the physical mechanism may be different.

Including both $M_{a}^{\text {tree }}$ and $M_{a}^{\text {anomaly }}$, the full expression for the gaugino masses upon moduli stabilization is given by [9]:

$$
M_{a} \approx\left[-0.032 \eta+\alpha_{\mathrm{GUT}}\left(0.034\left(3 C_{a}-C_{a}^{\prime}\right)+0.079 C_{a}^{\prime}(1-C)\right)\right] \times M_{3 / 2}
$$

where $C_{a}=(0,2,3)$ and $C_{a}^{\prime}=(33 / 5,7,6)$ for $\mathrm{U}(1)_{Y}, \mathrm{SU}(2)_{L}$ and $\mathrm{SU}(3)_{c}$ respectively. Here $1-\eta$ parameterizes threshold corrections to the $\mathrm{SU}(5)$ gauge coupling from Kaluza-Klein modes; as discussed in section $2.2,|1-\eta| \lesssim 0.1$ is expected for generic compactifications.

Thus taking $\eta \approx 1$, we have shown that top-down constraints from moduli stabilization fix the sparticle spectrum in terms of three almost-calculable constrained quantities: $M_{3 / 2}, \mu$, and $C$. Note that all SUSY breaking parameters in (A.6), (A.7) are implicitly defined at the renormalisation scale $Q=M_{\mathrm{GUT}}$. Given these "high scale" values, one must take into account RG-evolution and other radiative corrections to obtain sparticle pole masses as discussed in section 3 .

\section{B Constraints from EWSB and $M_{h}$}

In this appendix, we discuss how both EWSB and with consistency with $M_{h}=125.2 \pm$ $0.4 \mathrm{GeV}$ are imposed to reduce the dimensionality of the $M_{3 / 2}, \mu, C$ parameter space. We begin by consider the EWSB conditions, given by:

$$
\frac{M_{Z}^{2}}{2}=\frac{\bar{m}_{H_{d}}^{2}-\bar{m}_{H_{u}}^{2} \tan ^{2} \beta}{\tan ^{2} \beta-1}-\mu^{2}
$$




$$
B \mu=\frac{1}{2} \sin 2 \beta\left(\bar{m}_{H_{u}}^{2}+\bar{m}_{H_{d}}^{2}+2 \mu^{2}\right)
$$

where $\bar{m}_{H_{u}}$ and $\bar{m}_{H_{d}}$ are the tadpole corrected $H_{u}, H_{d}$ soft masses, and all parameters are evaluated at the renormalization scale $Q_{E W S B}^{2}=m_{\tilde{t}_{1}} m_{\tilde{t}_{2}}$. For the M-theory models we consider, we expect $\mu \lesssim 0.1 M_{3 / 2}$.

There are two independent arguments for such a suppression of $\mu$ with respect to $M_{3 / 2}$. The first is a top-down argument, which is related to the doublet-triplet splitting mechanism [20] discussed in section 2.2. This mechanism results in a geometric symmetry which forbids the $\mu$ term, which is broken by moduli stabilization to generate $\mu$ via the GiudiceMasiero mechanism [57]. Thus generically $\mu$ is suppressed with respect to $M_{3 / 2}$ by moduli vev's [21]. This suppression was estimated by [21] to be roughly an order of magnitude, though current theoretical uncertainties preclude a precise estimate. Another argument for $\mu \lesssim 0.1 M_{3 / 2}$ is motivated by electroweak naturalness. A measure of fine-tuning in EWSB is the degree to which the two terms on the right hand side of (B.1) are required to cancel in order to obtained the measured value of $M_{Z}$. Thus electroweak naturalness favors $\mu^{2} \ll M_{3 / 2}^{2}$, which mitigates the cancellation in (B.1) required to obtain $M_{Z} \approx 90 \mathrm{GeV}$ [25].

In addition to $\mu \lesssim 0.1 M_{3 / 2}$, we also expect $0<\bar{m}_{H_{u}}^{2} \ll \bar{m}_{H_{d}}^{2}$, as $m_{H_{u}}^{2}$ runs significantly between $Q=M_{\mathrm{GUT}}$ to $Q=Q_{E W S B}$ due to the top Yukawa coupling, while the running of $m_{H_{d}}^{2}$ is small for moderate $\tan \beta \lesssim 10$. The lower bound $0<\bar{m}_{H_{u}}^{2}$ is required for consistency with $\mu \lesssim 0.1 M_{3 / 2}$, as can be seen from (B.1). Taking the $B \mu, \bar{m}_{H_{d}}^{2} \gg \mu^{2}, \bar{m}_{H_{u}}^{2}$ and $\sin 2 \beta \approx 2 / \tan \beta$ limit, (B.1) can be written as [25]:

$$
\mu^{2} \approx \frac{\bar{m}_{H_{d}}^{2}}{B^{2}-\bar{m}_{H_{d}}^{2}}\left(\bar{m}_{H_{u}}^{2}+\frac{M_{Z}^{2}}{2}\right)
$$

where $B \approx 2 M_{3 / 2}$ at $Q=M_{\mathrm{GUT}}$ [8] and $B \approx 1.7 M_{3 / 2}$ at $Q=Q_{E W S B}$ [25]. Taking $\bar{m}_{H_{d}}^{2} \approx m_{0}^{2}$ and $\bar{m}_{H_{u}}^{2}=M_{3 / 2}^{2} f(C)$ where $f(C)$ accounts for the running of $m_{H_{u}}$ due to the top trilinear $A_{t}$, we can recast (B.3) in a more suggestive form:

$$
\mu^{2} \approx \frac{M_{3 / 2}^{2}(1-C)}{B^{2}-M_{3 / 2}^{2}(1-C)}\left(M_{3 / 2}^{2} f(C)+\frac{M_{Z}^{2}}{2}\right) .
$$

Note that $f(C)$ decreases monotonically as $C$ increases [25]. Thus we have used EWSB conditions to obtain a constraint on the allowed $M_{3 / 2}, \mu, C$ space; we use one-loop RGE's and one-loop effective potential corrections [58] to compute $f(C)$ for figure 1 . In order to minimize large logarithmic corrections, we run $\alpha_{s}$ and $y_{t}$ using a 2-step procedure which explicity accounts for the decoupling of squarks at $Q=Q_{E W S B}$.

We now discuss how the constraint on $M_{h}$ is incoporated in our analysis. The mass of the lightest Higgs boson in the MSSM decoupling limit is given schematically by:

$$
M_{h}^{2}=M_{Z}^{2} \cos ^{2} 2 \beta+\delta M_{h}^{2}
$$

where $\delta M_{h}^{2}$ denotes radiative corrections from both SM and MSSM particles; the value of $\delta M_{h}^{2}$ is fixed for given values of $M_{3 / 2}, \mu, C$. We can then use the EWSB condition (B.2) in 
the $B \mu, \bar{m}_{H_{d}}^{2} \gg \mu^{2}, \bar{m}_{H_{u}}^{2}$ and $\sin 2 \beta \approx 2 / \tan \beta$ limit to express $\tan \beta$ in terms $M_{3 / 2}, C$ and $\mu$ :

$$
\tan \beta \approx \frac{m_{0}^{2}}{B \mu} \approx \frac{M_{3 / 2}(1-C)}{B \mu}
$$

Thus combining (B.5) and (B.6) with $M_{h}=125.2 \pm 0.4 \mathrm{GeV}$, we obtain an additional constraint in the $M_{3 / 2}, \mu, C$ parameter space. To compute $M_{h}$ for given $M_{3 / 2}, \mu, C$, we use the "match-and-run" procedure, outlined for example in $[22,59]$. Note that some of the authors here made an error in [22] regarding SM radiative corrections to $M_{h}$; correcting this error increases $M_{h}$ by $\sim 1.5 \mathrm{GeV}$. Our calculation here uses 3-loop RGE's and 2-loop threshold corrections for the matching procedure [60]. From figure 1, we see that the slice of parameter space consistent with $M_{h}=125.2 \pm 0.4$ has a non-negligible width. This is due predominantly to experimental uncertanties in $M_{t}, \alpha_{s}$ and $M_{h}$ [22]; more precise measurements of these quantities will sharpen constraints on this parameter space.

\section{Threshold corrections from Kaluza-Klein states}

In this appendix we briefly discuss the Kaluza-Klein threshold corrections to the 7dimensional volume $V_{X}$. These corrections are parameterized in (2.3) by the topological invariant $L(\mathcal{Q})$, given by:

$$
L(\mathcal{Q})=4 q \sin ^{2}\left(\frac{5 \pi \omega}{q}\right)
$$

This form for $L(\mathcal{Q})$ was computed in [26], assuming that the visible sector $\mathrm{SU}(5)$ gauge fields are compactified on a Lens space $\mathcal{Q} \cong S_{3} / Z_{q}$ in the presence of a non-trivial Wilson line. $5 \omega$ is an integer parameterizing the effect of the Wilson line, as will be discussed below.

To proceed, we briefly review the motivation for considering $\mathrm{SU}(5)$ gauge theories compactified on a Lens space $\mathcal{Q}$. In $G_{2}$-compactifications of M-theory, non-abelian gauge fields arise from co-dimension $4 \mathrm{ADE}$ singularities and thus propogate on a 7-dimensional manifold $\mathcal{H}$ [2]. In our notation, we take $\mathcal{H} \cong \mathcal{Q} \times M$ where $M$ is our Minkowski spacetime. Starting from an SU(5) GUT theory, the issues of GUT breaking and doublet-triplet splitting must be resolved for a realistic model. As pointed out by Witten, both problems are elegantly solved in the presence of a non-trivial Wilson line background [20], which breaks $\mathrm{SU}(5)$ to the SM while admitting a geometric symmetry which solves doublet-triplet splitting.

The resulting symmetry is determined by the fundamental group of $\mathcal{Q}$, which for $\mathcal{Q} \cong$ $S / Z_{q}$ is simply $Z_{q}$. The non-trivial Wilson line gives the $\int d^{2} \theta \mu H_{u} H_{d}$ superpotential term charge $5 \omega$ under $Z_{q}$, while $\int d^{2} \theta M T_{u} T_{d}$ is uncharged. However, realistic phenomenology requires non-zero $\mu$, which implies that $Z_{q}$ is broken once moduli with charge $5 \omega$ obtain vev's [21]. If $Z_{q}$ is completely broken, higher-dimensional Kähler potential operators will generate dangerous lepton-number violating operators which generically violate neutrino mass bounds [16]. Thus $Z_{q}$ must be broken to a non-trivial subgroup $Z_{p}$, where:

$$
p=\operatorname{GCD}(q, 5 \omega), \quad p \neq 1
$$


The simplest case which satisfies these requirements is therefore the case where $q=4$ and $5 \omega=2$, corresponding to the Lens space $S_{3} / Z_{4}$ and an unbroken $Z_{2}$ symmetry once a non-zero $\mu$ term is generated.

Open Access. This article is distributed under the terms of the Creative Commons Attribution License (CC-BY 4.0), which permits any use, distribution and reproduction in any medium, provided the original author(s) and source are credited.

\section{References}

[1] G. Papadopoulos and P.K. Townsend, Compactification of D $=11$ supergravity on spaces of exceptional holonomy, Phys. Lett. B 357 (1995) 300 [hep-th/9506150] [INSPIRE].

[2] B.S. Acharya, M-theory, Joyce orbifolds and super Yang-Mills, Adv. Theor. Math. Phys. 3 (1999) 227 [hep-th/9812205] [INSPIRE].

[3] B.S. Acharya, On realizing $N=1$ super Yang-Mills in M-theory, hep-th/0011089 [INSPIRE].

[4] M. Atiyah and E. Witten, M-theory dynamics on a manifold of $G_{2}$ holonomy, Adv. Theor. Math. Phys. 6 (2003) 1 [hep-th/0107177] [INSPIRE].

[5] B.S. Acharya and E. Witten, Chiral fermions from manifolds of $G_{2}$ holonomy, hep-th/0109152 [INSPIRE].

[6] B.S. Acharya, K. Bobkov, G. Kane, P. Kumar and D. Vaman, An M-theory solution to the hierarchy problem, Phys. Rev. Lett. 97 (2006) 191601 [hep-th/0606262] [INSPIRE].

[7] B.S. Acharya, K. Bobkov, G.L. Kane, P. Kumar and J. Shao, Explaining the electroweak scale and stabilizing moduli in M-theory, Phys. Rev. D 76 (2007) 126010 [hep-th/0701034] [INSPIRE].

[8] B.S. Acharya, K. Bobkov, G.L. Kane, J. Shao and P. Kumar, The $G_{2}$-MSSM: an M-theory motivated model of particle physics, Phys. Rev. D 78 (2008) 065038 [arXiv:0801.0478] [INSPIRE].

[9] B.S. Acharya and K. Bobkov, Kähler independence of the $G_{2}-M S S M, J H E P 09$ (2010) 001 [arXiv:0810.3285] [INSPIRE].

[10] G. Kane, P. Kumar and J. Shao, CP-violating phases in M-theory and implications for EDMs, Phys. Rev. D 82 (2010) 055005 [arXiv:0905.2986] [INSPIRE].

[11] S.A.R. Ellis and G.L. Kane, Theoretical prediction and impact of fundamental electric dipole moments, arXiv:1405.7719 [INSPIRE].

[12] K. Kadota, G. Kane, J. Kersten and L. Velasco-Sevilla, Flavour issues for string-motivated heavy scalar spectra with a low gluino mass: the $G_{2}-M S S M$ case, Eur. Phys. J. C 72 (2012) 2004 [arXiv: 1107.3105] [INSPIRE].

[13] B.S. Acharya, K. Bobkov and P. Kumar, An M-theory solution to the strong CP problem and constraints on the axiverse, JHEP 11 (2010) 105 [arXiv:1004.5138] [INSPIRE].

[14] B.S. Acharya et al., Non-thermal dark matter and the moduli problem in string frameworks, JHEP 06 (2008) 064 [arXiv:0804.0863] [INSPIRE].

[15] G. Kane, J. Shao, S. Watson and H.-B. Yu, The baryon-dark matter ratio via moduli decay after Affleck-Dine baryogenesis, JCAP 11 (2011) 012 [arXiv:1108.5178] [INSPIRE]. 
[16] B.S. Acharya, G.L. Kane, P. Kumar, R. Lu and B. Zheng, R-parity conservation from a top down perspective, JHEP 10 (2014) 001 [arXiv: 1403.4948] [INSPIRE].

[17] G.L. Kane, P. Kumar, B.D. Nelson and B. Zheng, Dark matter production mechanisms with a non-thermal cosmological history - a classification, arXiv:1502.05406 [INSPIRE].

[18] J. Fan and M. Reece, In wino veritas? Indirect searches shed light on neutralino dark matter, JHEP 10 (2013) 124 [arXiv:1307.4400] [INSPIRE].

[19] T. Cohen, M. Lisanti, A. Pierce and T.R. Slatyer, Wino dark matter under siege, JCAP 10 (2013) 061 [arXiv: 1307.4082] [INSPIRE].

[20] E. Witten, Deconstruction, $G_{2}$ holonomy and doublet triplet splitting, hep-ph/0201018 [INSPIRE].

[21] B.S. Acharya, G. Kane, E. Kuflik and R. Lu, Theory and phenomenology of $\mu$ in M-theory, JHEP 05 (2011) 033 [arXiv: 1102.0556] [INSPIRE].

[22] G. Kane, P. Kumar, R. Lu and B. Zheng, Higgs mass prediction for realistic string/M-theory vacua, Phys. Rev. D 85 (2012) 075026 [arXiv:1112.1059] [INSPIRE].

[23] ATLAS collaboration, Measurement of the Higgs boson mass from the $H \rightarrow \gamma \gamma$ and $H \rightarrow Z Z^{*} \rightarrow 4 \ell$ channels with the ATLAS detector using $25 \mathrm{fb}^{-1}$ of pp collision data, Phys. Rev. D 90 (2014) 052004 [arXiv: 1406.3827] [INSPIRE].

[24] CMS collaboration, Precise determination of the mass of the Higgs boson and studies of the compatibility of its couplings with the standard model, CMS-PAS-HIG-14-009, CERN, Geneva Switzerland (2014).

[25] D. Feldman, G. Kane, E. Kuflik and R. Lu, A new (string motivated) approach to the little hierarchy problem, Phys. Lett. B 704 (2011) 56 [arXiv:1105.3765] [INSPIRE].

[26] T. Friedmann and E. Witten, Unification scale, proton decay and manifolds of $G_{2}$ holonomy, Adv. Theor. Math. Phys. 7 (2003) 577 [hep-th/0211269] [INSPIRE].

[27] I. Affleck, M. Dine and N. Seiberg, Dynamical supersymmetry breaking in supersymmetric QCD, Nucl. Phys. B 241 (1984) 493 [INSPIRE].

[28] D. Finnell and P. Pouliot, Instanton calculations versus exact results in four-dimensional SUSY gauge theories, Nucl. Phys. B 453 (1995) 225 [hep-th/9503115] [INSPIRE].

[29] B.S. Acharya, G. Kane and E. Kuflik, Bounds on scalar masses in theories of moduli stabilization, Int. J. Mod. Phys. A 29 (2014) 1450073 [arXiv:1006.3272] [InSPIRE].

[30] M. Papucci, K. Sakurai, A. Weiler and L. Zeune, Fastlim: a fast LHC limit calculator, Eur. Phys. J. C 74 (2014) 3163 [arXiv:1402.0492] [InSPIRE].

[31] B.C. Allanach, SOFTSUSY: a program for calculating supersymmetric spectra, Comput. Phys. Commun. 143 (2002) 305 [hep-ph/0104145] [INSPIRE].

[32] M. Muhlleitner, A. Djouadi and Y. Mambrini, SDECAY: a fortran code for the decays of the supersymmetric particles in the MSSM, Comput. Phys. Commun. 168 (2005) 46 [hep-ph/0311167] [INSPIRE].

[33] T. Moroi and L. Randall, Wino cold dark matter from anomaly mediated SUSY breaking, Nucl. Phys. B 570 (2000) 455 [hep-ph/9906527] [INSPIRE].

[34] B.S. Acharya, G. Kane, P. Kumar, R. Lu and B. Zheng, Mixed wino-axion dark matter in string/M-theory and the $130 \mathrm{GeV}$ gamma-line 'signal', arXiv:1205.5789 [INSPIRE]. 
[35] ATLAS collaboration, Search for strong production of supersymmetric particles in final states with missing transverse momentum and at least three b-jets using $20.1 \mathrm{fb}^{-1}$ of $p p$ collisions at $\sqrt{s}=8 \mathrm{TeV}$ with the ATLAS detector, ATLAS-CONF-2013-061, CERN, Geneva Switzerland (2013).

[36] ATLAS collaboration, Search for new phenomena in final states with large jet multiplicities and missing transverse momentum at $\sqrt{s}=8$ TeV proton-proton collisions using the ATLAS experiment, JHEP 10 (2013) 130 [Erratum ibid. 01 (2014) 109] [arXiv:1308.1841] [INSPIRE].

[37] CMS collaboration, Search for new physics in the multijet and missing transverse momentum final state in proton-proton collisions at $\sqrt{s}=8 \mathrm{TeV}$, JHEP 06 (2014) 055 [arXiv: 1402.4770] [INSPIRE].

[38] CMS collaboration, Search for supersymmetry in pp collisions at $\sqrt{s}=8 \mathrm{TeV}$ in events with a single lepton, large jet multiplicity and multiple b jets, Phys. Lett. B 733 (2014) 328 [arXiv: 1311.4937] [INSPIRE].

[39] J. Alwall et al., The automated computation of tree-level and next-to-leading order differential cross sections and their matching to parton shower simulations, JHEP 07 (2014) 079 [arXiv: 1405.0301] [INSPIRE].

[40] W. Beenakker, R. Hopker and M. Spira, PROSPINO: a program for the production of supersymmetric particles in next-to-leading order QCD, hep-ph/9611232 [INSPIRE].

[41] W. Beenakker, R. Hopker, M. Spira and P.M. Zerwas, Squark and gluino production at hadron colliders, Nucl. Phys. B 492 (1997) 51 [hep-ph/9610490] [INSPIRE].

[42] W. Beenakker et al., The production of charginos/neutralinos and sleptons at hadron colliders, Phys. Rev. Lett. 83 (1999) 3780 [Erratum ibid. 100 (2008) 029901] [hep-ph/9906298] [INSPIRE].

[43] CMS collaboration, Projected performance of an upgraded CMS detector at the LHC and HL-LHC: contribution to the Snowmass process, arXiv:1307.7135 [INSPIRE].

[44] T. Cohen et al., SUSY simplified models at 14, 33 and 100 TeV proton colliders, JHEP 04 (2014) 117 [arXiv:1311.6480] [INSPIRE].

[45] G.L. Kane, A.A. Petrov, J. Shao and L.-T. Wang, Initial determination of the spins of the gluino and squarks at LHC, J. Phys. G 37 (2010) 045004 [arXiv:0805.1397] [InSPIRE].

[46] H. Baer, V. Barger, A. Lessa, W. Sreethawong and X. Tata, Wh plus missing- $E_{T}$ signature from gaugino pair production at the LHC, Phys. Rev. D 85 (2012) 055022 [arXiv: 1201.2949] [INSPIRE].

[47] T. Cohen, R.T. D'Agnolo, M. Hance, H.K. Lou and J.G. Wacker, Boosting stop searches with a $100 \mathrm{TeV}$ proton collider, JHEP 11 (2014) 021 [arXiv:1406.4512] [INSPIRE].

[48] M. Low and L.-T. Wang, Neutralino dark matter at 14 TeV and $100 \mathrm{TeV}$, JHEP 08 (2014) 161 [arXiv: 1404.0682] [INSPIRE].

[49] S. Dawson, A. Ismail and I. Low, Redux on "when is the top quark a parton?", Phys. Rev. D 90 (2014) 014005 [arXiv: 1405.6211] [INSPIRE].

[50] B.S. Acharya, G. Kane and P. Kumar, Compactified string theories - generic predictions for particle physics, Int. J. Mod. Phys. A 27 (2012) 1230012 [arXiv:1204.2795] [InSPIRE]. 
[51] I. Affleck, M. Dine and N. Seiberg, Dynamical supersymmetry breaking in four-dimensions and its phenomenological implications, Nucl. Phys. B 256 (1985) 557 [INSPIRE].

[52] C. Beasley and E. Witten, A note on fluxes and superpotentials in $M$-theory compactifications on manifolds of $G_{2}$ holonomy, JHEP 07 (2002) 046 [hep-th/0203061] [INSPIRE].

[53] A. Brignole, L.E. Ibáñez and C. Muñoz, Soft supersymmetry breaking terms from supergravity and superstring models, Adv. Ser. Direct. High Energy Phys. 21 (2010) 244 [hep-ph/9707209] [INSPIRE].

[54] J.A. Bagger, T. Moroi and E. Poppitz, Anomaly mediation in supergravity theories, JHEP 04 (2000) 009 [hep-th/9911029] [INSPIRE].

[55] J.P. Conlon and F. Quevedo, Gaugino and scalar masses in the landscape, JHEP 06 (2006) 029 [hep-th/0605141] [INSPIRE].

[56] K. Choi and H.P. Nilles, The gaugino code, JHEP 04 (2007) 006 [hep-ph/0702146] [INSPIRE].

[57] G.F. Giudice and A. Masiero, A natural solution to the $\mu$ problem in supergravity theories, Phys. Lett. B 206 (1988) 480 [INSPIRE].

[58] D.M. Pierce, J.A. Bagger, K.T. Matchev and R.-J. Zhang, Precision corrections in the minimal supersymmetric standard model, Nucl. Phys. B 491 (1997) 3 [hep-ph/9606211] [INSPIRE].

[59] G.F. Giudice and A. Strumia, Probing high-scale and split supersymmetry with Higgs mass measurements, Nucl. Phys. B 858 (2012) 63 [arXiv:1108.6077] [INSPIRE].

[60] P. Draper, G. Lee and C.E.M. Wagner, Precise estimates of the Higgs mass in heavy supersymmetry, Phys. Rev. D 89 (2014) 055023 [arXiv: 1312.5743] [INSPIRE]. 